\title{
Una seconda opportunità per le cose. Circuito del dono e processi di mercificazione in un emporio dello scambio e del baratto
}

A second chance for things. Gift circuit and commodification process in an exchange and barter emporium

\section{Viola Lucrezia Giuliani}

\section{OpenEdition \\ Journals}

Edizione digitale

URL: http://journals.openedition.org/aam/1605

DOI: $10.4000 /$ aam. 1605

ISSN: 2038-3215

\section{Editore}

Dipartimento Culture e Società - Università di Palermo

Notizia bibliografica digitale

Viola Lucrezia Giuliani, « Una seconda opportunità per le cose. Circuito del dono e processi di mercificazione in un emporio dello scambio e del baratto ", Archivio antropologico mediterraneo [Online], Anno XXII, n. 21 (1) | 2019, online dal 30 juin 2019, consultato il 23 septembre 2019. URL : http:// journals.openedition.org/aam/1605; DOI : 10.4000/aam.1605

Questo documento è stato generato automaticamente il 23 septembre 2019

Archivio antropologico mediterraneo 


\title{
Una seconda opportunità per le cose. Circuito del dono e processi di mercificazione in un emporio dello scambio e del baratto
}

\author{
A second chance for things. Gift circuit and commodification process in an \\ exchange and barter emporium
}

Viola Lucrezia Giuliani

\section{Introduzione}

1 L'articolo che propongo è incentrato sull'analisi delle modalità di acquisizione e circolazione di oggetti in un emporio dello scambio e del baratto, il "Senza Monete", un'associazione senza scopo di lucro situata ad Orvieto, piccola cittadina umbra nella provincia di Terni. La ricerca, svolta a cavallo tra 2016 e 2017, si basa principalmente sull'osservazione partecipante e sulla realizzazione di interviste; l'interesse etnografico fa seguito alla mia frequentazione dell'associazione sin dalla sua fondazione quando, incuriosita dalla possibilità di scambiare oggetti che non reputavo più utili ed incoraggiata dalla condivisione dei principi etici di riciclo e riutilizzo che ispirano questo luogo, ho iniziato a partecipare al baratto.

2 Il fallimento e la sfiducia nelle strutture economiche e sociali che hanno investito l'Europa negli ultimi decenni, hanno spinto alcune comunità locali ad auto organizzarsi come reazione - e conseguenza - all'anomia culturale (Paltrinieri 1998), all'isolamento e alla frammentazione sociale: laddove il denaro scarseggia e la precarietà e l'incertezza sembrano dominare, il senso di comunità - fosse anche solo immaginata (Anderson 2018) -, il tempo libero, le competenze individuali e gli oggetti acquistano un rinnovato valore ed una riscoperta utilità. In tutta Europa sono sorti progetti e realtà associative che tentano di rispondere alla crisi attraverso strategie di innovazione economica, 
sociale e culturale che promuovono nuove forme relazionali grazie a reti di condivisione basate su innovative forme di produzione e consumo.

3 Ad Orvieto, l'associazione "Senza Monete. Emporio dello scambio e del baratto" offre una testimonianza di come la creatività di una piccola comunità abbia saputo fornire una risposta efficace alla crisi economica e sociale che negli ultimi anni ha spinto sempre più persone a cercare di soddisfare $\mathrm{i}$ propri bisogni materiali e relazionali attraverso metodi non convenzionali. Un approccio etnografico può aiutarci a comprendere meglio la densità e la complessità di queste dinamiche e reti sociali che, a tutti gli effetti, nascono come forme di protesta al capitalismo e di resilienza di fronte alla crisi.

\section{II "Senza Monete"}

L'associazione "Senza Monete. Emporio dello scambio e del baratto" nasce nel 2013, su iniziativa di alcuni soci della cooperativa sociale "Il Quadrifoglio". Lo scopo è dare vita ad un luogo dove le persone possano scambiarsi oggetti di ogni tipo, ad eccezione di cibi, bevande e medicinali. L'associazione ha sede all'interno dei locali dell'ex lavatoio comunale, concesso dal Comune di Orvieto in comodato d'uso, e si configura legalmente come associazione non profit gestita attraverso il volontariato. L'esperienza di Lorena Cupello, presidentessa dell'associazione, è stata determinante per l'ideazione e la realizzazione del progetto: lavorando sul territorio dell'orvietano come educatrice sociale, a contatto diretto con famiglie economicamente svantaggiate o in condizione di marginalità sociale, Lorena ha potuto osservare personalmente i bisogni materiali di molte persone che riscontrano difficoltà economiche quotidiane nell'acquistare beni attraverso il denaro. Queste osservazioni preliminari, sommate alla constatazione di quanti oggetti fossero stipati nei garage e nelle soffitte di molte case, in totale disuso, ha portato alla creazione di un luogo in cui rispondere sia alle situazioni di mancanza che a quelle di sovrabbondanza. Nel corso di un'intervista, Lorena afferma:

Questo progetto era nella mia mente già da un po' di tempo anche se non lo avevo ben definito. Sono un'educatrice sociale, lavoro per la psichiatria e frequento molte case in cui vedo tanti bisogni che necessitano di un supporto. Allo stesso tempo vedo altre situazioni in cui ci sono tante cose che non si usano più [...]. Quindi, associando queste due realtà, mi sono detta "perché non creiamo un posto dove chi può porta delle cose che magari non gli interessano più e invece chi ha necessità viene a vedere e se è interessato le prende".

5 L'associazione prevede una tessera soci annuale del costo di 5 euro, in cui sono inclusi 20 gettoni. Il gettone è la moneta di scambio utilizzata all'interno del "Senza Monete", l'unità di misura con cui viene valutato ogni oggetto che vi si trova e tramite il quale si può accedere al processo di scambio e di baratto. Non si tratta di un gettone materiale, bensì di una moneta simbolica, virtuale. Ogni oggetto portato all'interno dell'Emporio viene valutato dallo staff di volontarie su una scala che va da uno a cinque gettoni, come descritto dalla presidentessa:

È stato il direttivo a stabilire come valutare gli oggetti, è un discorso fittizio, valido solo all'interno della nostra attività. La valutazione va da 1 ad un massimo di 5, per esempio un vestito o un accessorio vale un gettone, le scarpe due gettoni, le coperte tre e così via. Ad ogni modo è un valore che è stato dato da noi in modo indicativo.

6 Tutto quello che viene portato al "Senza Monete" viene passato al vaglio dallo staff che ne valuta le condizioni e vi attribuisce un valore secondo il metodo sopra illustrato. I 
volontari si occupano di mantenere la contabilità attraverso registri in cui annotano, per ogni socio, la quantità di gettoni in entrata ed in uscita.

Più volte è stato ribadito che il baratto è l'unico modo di scambiare gli oggetti, come confermato dalla stessa Lorena: «Abbiamo voluto formulare così lo scambio: chi porta aumenta i suoi gettoni, chi prende li diminuisce ma tutto deve rimanere all'interno della formula di baratto, i gettoni non si possono comprare ma vanno guadagnati portando delle cose».

8 L'Emporio è aperto tre giorni a settimana ed è completamente gestito tramite il lavoro volontario di un gruppo di signore pensionate che si occupano della sua organizzazione, delle pulizie e della gestione degli oggetti scambiabili. Le volontarie sono affiancate da alcuni ragazzi e ragazze provenienti dalle cooperative sociali attive sul territorio che, in virtù del lavoro svolto, percepiscono una borsa lavoro terapeutica. Le Borse Lavoro sono state attivate per consentire a persone con particolare difficoltà di inserimento nel mondo lavorativo di accedervi gradualmente, attraverso un percorso di responsabilizzazione e socializzazione (Foto 1).

Il "Senza Monete" sorge in un'area periferica, ai piedi della rupe orvietana, al lato della stazione ferroviaria; in quest'area negli ultimi anni hanno visto la luce negozi e servizi di vario genere, nel tentativo di riqualificare uno spazio da sempre ai margini della vita sociale ed economica orvietana. L'Associazione ben si inserisce in questo progetto di riqualifica, non solo per i servizi offerti ma soprattutto per il tessuto sociale che si è venuto a creare con gli anni attorno alla comunità che lo gestisce. In particolar modo, nel tempo, sempre più ragazze e ragazzi provenienti dai centri di assistenza diurni per persone disabili hanno iniziato a collaborare settimanalmente nell'Emporio, aiutando sia nella gestione che nell'organizzazione del negozio. Il processo di responsabilizzazione delle persone che percepiscono le borse lavoro terapeutiche, mirato al raggiungimento di un certo grado di autonomia, non viene perseguito solo attraverso il lavoro, ma anche grazie alla possibilità di essere inseriti in un tessuto sociale inclusivo e accogliente.

La vicinanza con la stazione ferroviaria permette inoltre di raggiungere l'Associazione con una certa facilità: spesso ho incontrato in treno ragazzi e ragazze provenienti dai centri di accoglienza per richiedenti asilo, dislocati nelle campagne tra Chiusi e Orvieto che si recavano presso il "Senza Monete" alla ricerca di vestiti e scarpe. Frequentando i locali dell'Emporio, non solo durante i pomeriggi di rilevamento etnografico ma anche per effettuare personalmente gli scambi, ho potuto constatare il costante sforzo di creare un ambiente inclusivo soprattutto grazie alla collaborazione venutasi a creare tra i membri di questa comunità variegata ed eterogenea, uniti dal comune obiettivo di trovare sempre nuove strategie per il funzionamento dell'Emporio.

11 Nel corso degli anni il "Senza Monete" è stato oggetto di un'attenzione crescente sia da parte della popolazione del circondario orvietano sia di giornali e televisioni, locali e non, attratti dalla peculiarità del luogo. La comunità di soci che nel tempo si è venuta a creare attorno a questa realtà è spinta ad effettuare gli scambi per molteplici ragioni: c'è chi vi si reca alla ricerca di oggetti antichi o curiosi, chi spera di trovarvi vestiti vintage, chi sceglie di barattare vestiti e oggetti per questioni etiche o chi ha trovato nel "Senza Monete" la possibilità di sopperire a bisogni materiali trovandosi nell'impossibilità di acquistare prodotti tramite il denaro. Al di là delle ordinarie attività di scambio e baratto, le persone si recano presso i locali dell'Associazione per ritrovarsi con i soci e i volontari che abitualmente frequentano questo luogo. Non di 
rado capita di essere invitati a prendere parte ad una merenda improvvisata o a bere un caffè offerto dalle volontarie, scambiando quattro chiacchiere mentre si commentano i nuovi arrivi o mentre si sceglie un vestito o un oggetto.

Uno degli elementi di forza dell'Associazione risiede, quindi, nell'aspetto comunitario, capace di offrire una valida alternativa all'isolamento e alla marginalità, particolarmente percepito in certe fasce della popolazione escluse dalla vita economica e sociale orvietana. Se facciamo nostre le posizioni di Cnaan, Hunter e Milofsky (2005), si può affermare che una comunità si afferma quando vengono soddisfatte almeno tre condizioni principali: ecologica, che fa riferimento al tempo e allo spazio occupati; di struttura sociale, che ha a che vedere con le reti interpersonali e la densità istituzionale al loro interno; e simbolico-culturale, laddove vi siano un'identità e una cultura comuni. Quando tutti e tre questi fattori sono combinati, si è in presenza di una forte comunità (Taylor 2010: 237). Nello specifico del "Senza Monete" questi tre elementi sembrano essere presenti nel creare una realtà che tenta di colmare le carenze delle politiche pubbliche e sociali attraverso un'alternativa economica e comunitaria basata sulla condivisione di valori e obbiettivi comuni. Ovviamente, possiamo considerare la comunità anche come l'effetto di un processo immaginato, dove le persone si ritrovano insieme perché condividono desideri e aspirazioni ancora prima di un tessuto socioculturale comune (Anderson 2018; Appadurai 2012). O, in ultima istanza, come comunità di pratica (Grasseni, Ronzon 2004).

La singolarità di questa realtà associativa ha attratto l'attenzione di molte associazioni nazionali che, ispirate dal funzionamento e dal successo dell'Emporio, hanno pensato di esportare questo progetto anche in altri luoghi della Penisola. Inoltre, le caratteristiche di questa associazione sono state negli anni fonte di ispirazione per studiosi di vari settori quali economisti, antropologi, sociologi, assistenti sociali che vedono nel "Senza Monete" un concentrato di elementi particolarmente interessanti da esaminare ed approfondire.

\section{Gli oggetti scambiabili}

14 L'osservazione degli scambi e degli oggetti presenti all'interno dell'Emporio permette di trovare numerosi nessi con alcune teorie delle merci in antropologia, ispirate a vario titolo dalla definizione marxiana di merce - in particolare quelle di Appadurai (1986, 2014), Kopytoff (1986), Weiner (1992, 1994). La definizione marxiana di merce come oggetto esterno in grado di soddisfare i bisogni umani e la cui utilità si definisce nel valore d'uso, ha avuto ampia risonanza nelle scienze sociali, influenzando gli sviluppi dell'antropologia economica e della cultura materiale. L'insistenza dell'approccio marxista sulla produzione e sui meccanismi di riproduzione delle classi sociali non lascia però molto spazio alla trattazione della libertà dei soggetti in merito alle scelte di consumo.

15 I "nuovi studi" di cultura materiale, invece, integrano l'approccio marxista con un'analisi ed una teoria delle merci più complessa ed etnografica, grazie ad un'attenta riflessione sulla vita sociale degli oggetti e ad un nuovo interesse verso pratiche di consumo precedentemente non indagate (Dei, Meloni 2015: 55). Sebbene nelle prime indagini etnografiche, concentrate principalmente sullo studio delle società preindustriali, le pratiche di consumo siano in qualche misura analizzate e descritte, è a partire dagli anni Sessanta e Settanta del Novecento che queste diventano un campo di 
primo interesse per l'antropologia. Dagli anni Ottanta ad oggi il consumo è stato uno dei campi indagati con più versatilità e ha consentito agli antropologi di concentrarsi sia sulle pratiche delle società occidentali contemporanee, di stampo industriale e capitalista, sia sulle mutazioni delle società postcoloniali (Torsello 2007; Miller 1987, 2012). Gli studi contemporanei incentrati sulle pratiche di consumo sono caratterizzati da posizioni diverse e talvolta contrastanti. La critica nei confronti della globalizzazione dei mercati, dell'omologazione dei consumi e dei rapporti di dipendenza tra Paesi nello scenario politico ed economico postcoloniale tende a porre l'accento sulla passività dei consumatori, sui rapporti di potere tra gli Stati e sui meccanismi che generano gli squilibri economici globali.

Una posizione diversa è espressa da autori come Daniel Miller che, attraverso un'attenta etnografia del mondo contemporaneo, consentono di analizzare come gli attori sociali si approprino del mondo delle merci, conferendo loro significato all'interno del proprio orizzonte di senso: soggetti dotati di agency che operano delle scelte razionali e motivate (Meloni 2018). Un'etnografia della vita quotidiana, come quella proposta in Teoria dello shopping (Miller 1998) consente di riflettere sulla costruzione di identità e legami sociali che vengono prodotti e riprodotti attraverso un'attività apparentemente banale come la spesa quotidiana: una pratica che, grazie a questa chiave di lettura, non risulta irrazionale o edonistica, ma al contrario attentamente calcolata, altruistica e ritualizzata.

17 Se l'analisi delle quotidianità permette di cogliere l'agency degli attori sociali nell'appropriarsi degli oggetti e nel costruire o rimarcare i loro legami affettivi e sociali, autori come Kopytoff e Appadurai aiutano a spostare l'attenzione sulla dimensione sociale e dinamica delle merci, ponendo l'accento sulla «inseparabilità antropologica del "sociale" e del "materiale"» (Dei, Meloni 2015: 57). Kopytoff in The cultural biography of things (1986) propone un'efficace analogia tra la schiavitù, suo principale campo di indagine, e le merci per dimostrare come le traiettorie di vita e i contesti sociali e culturali possano portare le cose, così come le persone, dentro e fuori lo stato di merce. L'analisi prende le mosse dalla constatazione di come, nella società occidentale, si tenda a ricondurre unicamente gli oggetti all'universo della mercificazione mentre le persone appartengono all'universo opposto, definito dall'autore della "singolarizzazione". La schiavitù sovverte questa dicotomia: sono gli esseri umani ad essere ridotti allo stato di merci, scambiabili e possedibili. Kopytoff propone di analizzare le traiettorie di vita degli oggetti, attraverso la loro "biografia culturale", così da ricostruire i contesti e le traiettorie che sono alla base della costruzione della merce.

Questa operazione permette di notare come lo status di merce non sia una condizione perenne e intrinseca alle cose: un oggetto può acquisire nel corso della sua vita delle caratteristiche che lo demercificano completamente, talvolta sacralizzandolo. Allo stesso tempo un oggetto, in un primo momento protetto dalla possibilità di essere scambiato e mercificato, può essere inserito in un circuito di scambio che ne muta lo status. La mercificazione e l'alienabilità (così come la possibile demercificazione e inalienabilità) non risultano più delle caratteristiche immutabili, proprie delle cose, ma degli attributi riconducibili a certe condizioni della loro vita sociale, in un determinato contesto storico, culturale e sociale.

19 Una differente - se non opposta - posizione è espressa da Gregory in Gift and Commodities (1982). L'autore, attraverso un'attenta e dettagliata etnografia della società 
e dell'economia della Papua Nuova Guinea, propone una rilettura delle teorie economiche e antropologiche a lui precedenti, individuando nel dono e nella merce due sistemi distinti, talvolta coesistenti e caratterizzanti contesti sociali e culturali differenti. Nell'affermare ciò l'autore asserisce che l'economia del dono e l'economia delle merci dovrebbero essere viste come due possibili sistemi economici in cui il dono e la merce si configurano come le due distinte categorie caratterizzanti tali sistemi. Stando a quanto afferma Gregory verrebbe da chiedersi: cosa rende, quindi, un oggetto candidabile come merce? E cosa lo differenzia dal dono? Appadurai, per quanto affascinato dal discorso sui processi di mercificazione proposti da Gregory - come anche da quelli di Taussig (2017) - prende le distanze dall'eccessiva enfasi sul contrasto tra economia del dono ed economia della merce (Appadurai 2013: 80). Egli propone di pensare, sulla scia della mercificazione di Hart (1982), che tende a riavvicinare Marx a Mauss, la condizione di merce nella vita sociale di una qualsiasi cosa come il momento in cui «la sua scambiabilità (passata, presente o futura) con qualche altro oggetto sia il suo elemento socialmente rilevante» (ivi: 29). Le merci non risultano più delle classi particolari di cose, ontologicamente differenziate dal dono, bensì degli oggetti in condizioni specifiche, in determinati momenti della loro vita (Foto 2).

Il "Senza Monete" si presenta come un caso paradigmatico che permette di osservare e ricostruire le traiettorie di vita di una vasta gamma di oggetti. Passeggiando tra gli scaffali espositivi dell'Emporio capita spesso di imbattersi in piccole suppellettili che richiamano l'attenzione: oggetti curiosi, carichi di storia, che suggeriscono all'immaginazione di chi li osserva di tentare di ricostruire la loro vita passata o di proporsi come possibili candidati per un nuovo capitolo della loro esistenza sociale. Sebbene caratterizzati da diverse biografie culturali, una volta collocati sugli espositori del "Senza Monete", tutti gli oggetti sono considerati delle merci: cose potenzialmente scambiabili, a prescindere dalla transazione con cui vengono fatte circolare. Se nell'Emporio la principale caratteristica degli oggetti risiede nella loro scambiabilità è lecito chiedersi se tra gli espositori vi siano anche cose fino a quel momento protette dalla possibilità di essere immesse in un circuito di scambio in virtù del valore $\mathrm{e}$ dell'importanza che le legava a chi le possedeva precedentemente.

21 Weiner parla di "densità degli oggetti" (1994) portando a riflettere sul processo e sulle caratteristiche che trattengono alcuni oggetti dall'essere scambiati entrando in un circuito di reciprocità e alienabilità. Oggetti densi di valori e significati che difficilmente possono essere separati dai loro proprietari e che in virtù della loro densità resistono allo scambio e sfuggono alla logica del dare, ricevere e ricambiare. Nel "Senza Monete" gli oggetti, per quanto in passato possano essere stati carichi di valori, attribuitigli dalle vicende della loro biografia culturale, sono inseriti in un circuito di scambio che li rende alienabili e soggetti ad un processo di mercificazione che ne annulla o ne modifica la densità acquisita nel tempo.

Chi entra per la prima volta nell'Emporio può avere l'impressione di trovarsi presso la bottega di un rigattiere o in un negozio di vestiti usati. Gli oggetti esposti sugli scaffali al piano terra vanno da piccoli soprammobili e suppellettili a vecchie lampade, servizi di piatti, libri o piccola bigiotteria. Un'intera parete è dedicata agli accessori per la casa: posate e set di piatti che richiamano alla memoria l'arredamento della casa di una vecchia zia, elettrodomestici non sempre funzionanti, vassoi dalle fantasie floreali, tovaglie di pizzo, tende ricamate all'uncinetto, portatovaglioli in legno o in plastica lucida, bicchieri dal vetro un po' opaco, consumato dall'uso. Il primo piano è invece 
interamente dedicato a vestiti e scarpe per uomo, donna e bambino. I vestiti da donna e da bambino sono i più numerosi e i più richiesti. Talvolta è possibile trovarvi vestiti cuciti a mano o di marche particolarmente prestigiose. Gli appassionati di moda vintage vi si recano alla ricerca di pezzi unici, magari dimenticati per anni negli armadi o custoditi in vecchi bauli in qualche soffitta.

La quantità di oggetti curiosi che è possibile trovarvi è una delle principali attrazioni per molti dei soci, come testimoniato da una signora nel corso di un'intervista: «Sono un'appassionata di oggettistica e qui ho la possibilità di trovare cose particolari, come quelle che si trovano nei mercatini dell'usato, ma qui non le devo pagare» (Foto 3). Un'attenta analisi degli oggetti esposti nell'Emporio permette di osservare come essi appartengano a categorie estremamente eterogenee: vi sono beni di produzione industriale e seriale e prodotti artigianali. Inoltre, è possibile trovarvi numerosi manufatti come calze fatte all'uncinetto, disegni realizzati a mano, fotografie d'epoca o raffigurazioni religiose.

24 Appadurai (2013) propone di analizzare le merci attraverso quattro categorie, focalizzando l'attenzione sulle traiettorie di vita e sulle finalità con cui tali merci sono state pensate, progettate e fatte circolare: merci per destinazione, per metamorfosi, per deviazione, ex merci. La maggior parte degli oggetti che si trovano all'interno dell'Emporio sono «merci per destinazione, vale a dire oggetti intesi dai loro produttori principalmente per lo scambio» (Appadurai 2013: 33): oggetti di produzione industriale, pensati e prodotti per essere mercificati.

Curiosando tra gli scaffali del "Senza Monete" capita spesso di imbattersi in articoli che non ci si aspetterebbe di trovare su un espositore, destinati ad essere scambiati. Durante uno dei pomeriggi in cui ho svolto il rilevamento etnografico ho osservato a lungo un disegno fatto a mano con i colori a tempera, un disegno dalle pennellate un po' incerte, probabilmente realizzato da una bambina, raffigurante una signora seduta su una poltrona marrone. Ho ipotizzato che si trattasse di un esempio di «merci per metamorfosi, oggetti intesi per altri usi posti nello stato di merci» (ivi: 33). Non potendo conoscere le traiettorie che hanno portato gli oggetti nell'Emporio, rendendoli disponibili allo scambio, è difficile sapere se si è in presenza di una merce per deviazione, ossia di «oggetti messi nello stato di merci benché all'origine siano specificamente protetti da questa eventualità» (ibidem). L'analisi degli oggetti scambiabili permette di notare come potenzialmente tutto ciò che si trova esposto nei locali dell'Associazione sia considerabile una merce, se per merce intendiamo «qualsiasi cosa finalizzata allo scambio» (ivi: 23). Tutti gli oggetti contengono in sé un potenziale, una possibilità latente di essere scambiati, mercificati o demercificati. Per essere analizzati in quest'ottica devono, tuttavia, essere dotati «delle stesse forme di agency, di energia e di accadimento biografico che attribuiamo a noi stessi» (ivi: 411).

\section{Portare, prendere, scambiare}

L'associazione "Senza Monete" conta diverse centinaia di soci provenienti da tutto il circondario orvietano, persone di tutte le età, nazionalità e classi sociali, spinte da molteplici motivazioni a partecipare al baratto. Durante la ricerca ho avuto la possibilità di intervistare una decina di soci, con lo scopo di comprendere le ragioni che spingono a partecipare agli scambi e a scegliere questa forma di "economia alternativa". Le motivazioni che sono emerse sono in parte testimonianza di alcune 
riflessioni teoriche sul consumo e sullo spreco, in parte conseguenza di disuguaglianze e difficoltà economiche. Molte delle persone che si recano al "Senza Monete" per portare o prendere oggetti sono mosse dalla volontà di abbattere o ridurre gli sprechi e di partecipare ad una pratica alternativa al consumismo dando una nuova destinazione agli oggetti e ai vestiti, come testimoniato dalle parole di una signora che afferma:

Oggi ho portato delle scarpe, me le aveva regalate un'amica ma sinceramente non mi sono mai piaciute allora le ho portate qui. Mi piace fare scambi. Vengo, porto qualcosa, prendo qualcosa, o magari non prendo niente se non c'è niente che mi piace. [...] Trovo che sia molto bello quello che fanno qui, dovrebbe essere fatto di più. Alla fine, è un'alternativa al consumismo, no? Io lo faccio anche con le mie amiche, ci scambiamo sempre le cose. Il baratto è un principio che condivido, mi piace.

Questa impostazione, etica e critica al contempo, è emersa in molte delle conversazioni sostenute con le frequentatrici e i frequentatori dell'Emporio, come sottolineato da un'altra signora intervistata: «Secondo me è una soluzione allo spreco. Sprechiamo un sacco di cose, no? Almeno qui si possono prendere, riutilizzare».

Le posizioni etiche che sono alla base delle scelte di questa componente dei soci trovano riscontro in molte pratiche di consumo alternativo, oggi sempre più diffuse, e sono supportate da una nutrita letteratura, fortemente critica con il consumo e il consumismo (Latouche 2008, Bauman 2010). Il concetto di "decrescita serena" di Latouche, ad esempio, è citato nei volantini informativi dell'associazione, sulla sua pagina internet e nelle testimonianze dei fondatori di questa realtà, a conferma di come l'ideazione e la realizzazione del "Senza Monete" sia stata ispirata da una chiara ed espressa posizione teorica. Se «l'economia consumistica deve fare affidamento sull'eccesso e sullo spreco» (Bauman 2010: 49), realtà come il "Senza Monete" propongono una interessante riflessione sulle strategie messe in atto da comunità di consumatori, più o meno numerose e organizzate, di creare dei circuiti in cui gli oggetti possano continuare a circolare, trovando nuove destinazioni e riscoperte utilità.

Alcune delle interviste sono state realizzate nei giorni precedenti le festività natalizie. L'afflusso di soci era notevole e per l'occasione era stata ideata una lotteria con in palio alcuni oggetti selezionati dalle volontarie per la loro rarità e per il valore estetico $\mathrm{e}$ monetario che gli veniva attribuito. Alcuni dei soci che si sono dimostrati disponibili a rispondere alle domande hanno dichiarato di trovarsi nell'Emporio per cercare regali di Natale per amici e familiari, come affermato da una ragazza, assidua frequentatrice dell'associazione:

Sto cercando dei regali di Natale e visto che avevo un po' di tempo sono venuta qui [...] Mi rilasso, guardo i vestiti, gli oggettini, è come una boutique solo che non ci sono le commesse a metterti pressione, a farti venire l'ansia. Poi mi piace che qui non ci sono i soldi di mezzo, insomma secondo me è anche terapeutico perché $\mathrm{i}$ soldi mettono ansia.

Così come nei negozi o nei centri commerciali non è raro incontrare persone che vi si recano per "rilassarsi" guardando le vetrine o facendo shopping, anche nel "Senza Monete" è possibile incontrare persone di tutte le età che passeggiano tra gli scaffali espositivi, talvolta senza portare a casa alcunché. Bauman (2010) osserva come la società consumistica incoraggi un tipo di atteggiamento che catalizza in un consumo irrazionale e compulsivo una serie di bisogni, ansie e insoddisfazioni, che sembrano trovare una soluzione nelle vetrine dei negozi e nella sfrenata corsa all'acquisto. L'immagine della commessa opprimente che cerca di invogliare il cliente a comprare, 
vendendo una merce potenzialmente superflua come assolutamente necessaria ci restituisce un'immagine in carne e ossa di un'economia che mira all'irrazionalità del consumatore, facendo leva sulle sue «emozioni consumistiche» (Bauman 2010: 60).

Al "Senza Monete" nessuno fa pressione affinché un socio esca dall'Emporio con una giacca o un soprammobile nuovo. Il fatto che non vi sia profitto economico per l'associazione fa sì che in molti vivano il consumo con una maggiore rilassatezza: nell'Emporio ogni acquisto potenziale si traduce nella spesa massima di cinque gettoni per ogni singolo pezzo. Nell'intervista viene poi messa in evidenza l'ansia suscitata dal denaro ed il potenziale "terapeutico" di uno shopping senza transazioni monetarie; si viene qui a creare un paradosso tra le necessità create da una società del consumo ed una soluzione considerata alternativa al consumismo. Dalle parole della ragazza emerge come le ragioni che spingono a recarsi al "Senza Monete", anche solo per passeggiare tra gli scaffali espositivi, siano le stesse che motivano uno shopping compulsivo, immotivato e superfluo; la differenza tra questi due atteggiamenti non risiede nelle motivazioni di fondo che inducono all'acquisto ma nelle soluzioni pratiche messe in atto e nelle giustificazioni date a se stessi e agli altri a sostegno del proprio atteggiamento.

Stando a quanto emerge dalle parole della ragazza il denaro sembra essere in un certo senso "demonizzato", non solo in quanto fonte di ansia ma anche per il suo potenziale discriminatorio: recarsi in un negozio, osservare l'oggetto del proprio desiderio senza poterlo acquistare per mancanza di possibilità economiche produce nel consumatore un senso di inadeguatezza e inappagamento. Per Hart questa demonizzazione del denaro non tiene conto del valore che esso ha in quella che definisce human economy (Hart 2009: 99). Il denaro non dovrebbe essere interpretato solo in virtù dell'ineguaglianza che produce e per il suo potere di sfruttamento, ma andrebbe considerato anche per le sue proprietà redentive, come mediatore tra l'individuo e la società: lo strumento attraverso il quale umanizzare la società, permettendo all'individuo di stabilizzare la sua identità, concretizzando i suoi desideri in qualcosa di duraturo (ivi: 92) (Foto 4).

Vi è poi una vasta componente di soci che si rivolge all'associazione per soddisfare bisogni materiali, trovandosi senza reddito o in difficoltà economica. Una socia, incontrata in più di un'occasione spiega così la sua frequentazione dell'Emporio:

Vengo qui anche perché non posso spendere e grazie a questo posto ho trovato tante cose, mi ci sono rivestita [...] non vivo bene, vivo con 400 euro al mese che non mi permettono di andare avanti [...] Qualche volta, non sempre, qui trovo cose che sono veramente buone [...] Ho sempre rispettato gli altri perché so che qui ci vengono persone che hanno veramente bisogno, persone che non hanno neanche reddito.

34 Le diseguaglianze sociali ed economiche che caratterizzano il nostro Paese (Franzini 2013) spingono sempre più persone a rivolgersi a cooperative o ad associazioni assistenziali che aiutano nel soddisfacimento dei bisogni materiali di quella fascia della popolazione considerata al di sotto della soglia di povertà.

Gli esempi fin qui riportati ci permettono di riflettere sull'eterogeneità dei consumatori: parte dei soci vi si reca per divertimento e per curiosità, mentre una componente consistente è spinta da necessità. Tuttavia, il meccanismo che regola il "Senza Monete" permette a chi vi si rivolge di partecipare ad una transazione che non crea la sensazione di ricevere la carità: il baratto genera parità, un dare ed avere che 
pone tutti nella stessa posizione, permettendo un'uscita, per lo meno simbolica e momentanea, dalla deprivazione materiale e dalla povertà.

\section{Donare tempo, donare cose}

La maggior parte degli scambi che avvengono all'interno dell'Emporio sono regolati dalla logica del baratto descritta in precedenza: i soci portano oggetti che, una volta convertiti in gettoni, consentono di prendere e portare a casa vestiti, scarpe o suppellettili per un valore complessivo uguale a quello degli oggetti portati. Nei giorni in cui ho effettuato le interviste e ho osservato le transazioni dei soci, ho potuto riscontrare alcune forme di scambio che si discostano dal baratto e che richiamano più o meno esplicitamente la logica del dono. Alcuni dei frequentatori del "Senza Monete" si dimostrano più propensi a portare che a prendere: molte persone entrano con borse piene di vestiti ed oggetti che lasciano presso l'Emporio dichiarando di non voler prendere alcunché con i gettoni guadagnati. Questi gettoni rimangono come "sospesi" e, nell'attesa di essere utilizzati, vengono messi a disposizione delle persone che non possono partecipare al baratto in quanto non possiedono o non portano niente da scambiare.

Negli ultimi anni molte persone provenienti dai centri di accoglienza per richiedenti asilo situati nella zona dell'orvietano si recano nell'Emporio per prendere vestiti e scarpe; si tratta di persone che non partecipano al baratto e che utilizzano i gettoni messi a disposizione dai soci, usufruendo del servizio offerto dal "Senza Monete" come se si trattasse di un'associazione assistenzialista e filantropica. Dalle conversazioni con le volontarie è emerso che numerose famiglie del circondario orvietano, che vivono situazioni di particolare fragilità sociale e difficoltà economica, si rivolgono all'Emporio per sopperire ad alcuni bisogni materiali senza partecipare al baratto, anch'essi usufruendo di questo servizio come fosse un'associazione caritatevole. Durante una lunga conversazione, due volontarie mi hanno spiegato i meccanismi con cui si cerca di far fronte a queste particolari situazioni di difficoltà, nel tentativo di fornire un aiuto materiale concreto ed immediato. Ad esempio, alcuni oggetti utili e rari, come interi servizi di piatti o piccoli elettrodomestici ancora funzionanti, vengono tolti dal circuito di scambio e destinati direttamente a persone che ne hanno dichiarata necessità; le volontarie stesse si fanno carico del sostegno alle famiglie ed ai soggetti più vulnerabili che si rivolgono all'Associazione confortati e incoraggiati dalla solidarietà del gruppo che si è costituito negli anni attorno all'Emporio.

Il tipo di transazione finora descritto esula completamente dalla logica del baratto ed aderisce maggiormente a quella del dono. Un dono che in un primo momento avviene tra sconosciuti, tra soggetti che non necessariamente si conosceranno o si incontreranno: un dono dai costi sociali limitati, se non nulli, che permette a chi dona di manifestare un altruismo che va oltre la rete dei propri rapporti sociali (Godbout 2002: 83). La circolazione degli oggetti donati nel caso del "Senza Monete", rappresentati dai gettoni sospesi o dai prodotti tolti dal circuito di scambio, avviene poi ad opera e per merito delle volontarie, grazie alla rete di rapporti interpersonali intessuti negli anni con la popolazione locale. Se interpretiamo questa forma di circolazione degli oggetti attraverso il paradigma del dono teorizzato da Mauss, suddiviso nei tre momenti del dare, ricevere e ricambiare, notiamo come il terzo momento venga meno: la transazione prevede due soggetti, chi dona e chi riceve: il 
secondo soggetto non è chiamato a ricambiare, non contrae alcun obbligo, gli viene richiesto unicamente di ricevere. Lo stesso Mauss afferma che «il dono non ricambiato rende [...] inferiore colui che lo ha accettato, soprattutto quando accolto senza l'intenzione di restituirlo» (Mauss 2002: 117).

La gerarchia di poteri che si viene a creare nelle attività filantropiche e solidaristiche delinea un netto confine tra due gruppi: i donatori e i riceventi. L'impossibilità di ricambiare il dono congela i due status, rimarcando la superiorità materiale e simbolica del primo gruppo sul secondo. L'assenza di costi sociali tra donatore e ricevente è un'ulteriore caratteristica di questo tipo di transazione: il dono così descritto non crea né fortifica i legami sociali tra il soggetto donatore e quello ricevente. Tuttavia, nel caso concreto del "Senza Monete" le volontarie, che fungono da intermediarie tra chi dona e chi riceve, si fanno carico dei costi sociali dei doni, creando una rete di rapporti umani e personali all'interno della comunità costituitasi negli anni attorno all'Associazione.

Ma torniamo ai donatori. Se il loro donare non crea legami sociali e non prevede alcun tipo di tornaconto materiale, ci si potrebbe chiedere: perché donare? In questa forma di dono unilaterale a degli sconosciuti possiamo riscontrare molte caratteristiche tipiche della beneficenza: la motivazione del dono risiede nello stesso donare, in quanto «c'è una restituzione immediata di energia per colui che dona, il quale ne esce ingrandito» (Godbout 2002: 123). La percezione di "fare qualcosa di buono" attraverso un semplice atto crea nel donatore una immediata sensazione di accrescimento e benessere personale.

41 Lo stesso tipo di trasformazione che avviene nel donatore è riscontrabile anche nel volontariato. Se analizzato in chiave utilitaristica, il volontariato altro non è che un'attività lavorativa non remunerata $\mathrm{o}$, in altre parole, una forma di dono che si traduce in servizi. Che cosa guadagna quindi un volontario per la sua attività? Chi fa volontariato afferma di ricevere molto in cambio del suo impegno e del suo tempo, come testimoniato da una delle volontarie del "Senza Monete" che dichiara «ricevo tante soddisfazioni dalle persone che capiscono questo sistema [...] stiamo bene qui, tra di noi, siamo tutti compatibili».

42 Appare evidente come la restituzione di cui si parla in questo ambito non avvenga nel senso materiale e mercantile del termine, ma si riferisca ad un senso di soddisfazione e di partecipazione ad una causa comune, avvertita come più importante e remunerativa di un qualsiasi compenso materiale. La maggior parte delle persone che si dedicano ad attività di volontariato afferma di ricevere in cambio molto più di quanto venga da loro dato: da una parte il senso del dovere guida l'attività volontaria, dall'altra il piacere e la libertà d'azione caratterizzano questo tipo di lavoro (ivi: 100).

Anche nel volontariato il terzo momento della tripartizione maussiana del dono sembra venire meno: la stessa attività volontaristica appare unidirezionale e generatrice di gerarchie e rapporti asimmetrici, perché anche qui il dono è orientato in un solo senso, non è accettato alcun ritorno, non c'è azione di reciprocità (Coluccia 2002: 29). Tuttavia, se invertiamo la prospettiva potremmo dire che nel volontariato i tre momenti del dono maussiano si confondono poiché «secondo il punto di vista dell'attore, donare è ricambiare e viceversa» (Godbout 2002: 124). La distinzione tra donare e ricambiare sembra essere quindi puramente analitica: da parte di chi si dedica ad attività di volontariato il suo stesso impegno è già un ricambiare: una sorta di restituzione, libera e volontaria, di quanto si è già ricevuto dalla vita. Il donare è quindi un ricambiare. 
Nell'analisi dell'organizzazione e delle transizioni che avvengono nel "Senza Monete" sono emerse, poi, ulteriori dinamiche peculiari che richiamano apertamente la logica del dono. Una volontaria con cui ho avuto una lunga conversazione sul funzionamento e la gestione dell'Emporio mi ha spiegato che alcuni negozianti donano all'associazione le rimanenze di magazzino o i vestiti e le scarpe della stagione precedente. Si tratta per lo più di persone solidali con il progetto del "Senza Monete", che supportano il lavoro dei volontari attraverso i loro donativi, permettendo così ai soci di portare a casa vestiti o oggetti nuovi e mai utilizzati. Anche questi doni appaiono come totalmente gratuiti e svincolati da qualsiasi logica utilitaristica: i negozianti non si aspettano alcun tipo di controdono né pretendono che il loro gesto sia in qualche modo contraccambiato. Nuovamente la logica del dono teorizzata da Mauss sembra interrompersi proprio nel momento in cui la controparte dovrebbe rispondere. Come nel volontariato o nel caso dei gettoni lasciati "sospesi" dai soci, si insinua nuovamente la domanda: "sono davvero gratuiti questi doni"?

Mary Douglas risponderebbe che:

Non ci sarebbe dovuto essere un solo dono al mondo, se si fosse presa alla lettera questa condizione di gratuità. L'errore del cosiddetto dono gratuito o disinteressato è l'attribuire al donatore l'intenzione di non avere indietro nessun controdono dal ricevente. Ma rifiutando ogni possibilità di reciprocità, pone l'atto del donare fuori dal suo contesto sociale e lo priva di qualsiasi significato relazionale. Si sostiene che una volta donato, il dono libera da ogni obbligazione e non implica ulteriori richieste da parte di chi lo riceve (Douglas 2007: 405).

La nozione di gratuità sottintende l'unilateralità assoluta: in una logica puramente utilitaristica e mercantile il dono gratuito semplicemente non esisterebbe, in quanto nella gratuità sono assenti sia l'equivalenza calcolata sia la logica del profitto; sostituendo l'obbligo con la libertà potremmo spiegare il paradosso apparente che si viene a creare nel dono gratuito: chi dona accetta tacitamente la perdita, mosso dal piacere di donare. La gratuità del dono non implica una sottovalutazione o una totale inconsapevolezza del calcolo o della perdita: gli agenti, laddove agiscono consapevolmente, rifiutano coscientemente una qualsiasi ricompensa materiale. Eppure, il vero problema non risiede tanto in chi dona ma in chi riceve: non contraccambiare il dono implica la contrazione di un debito permanente, un'esclusione dal legame sociale paritario ed equilibrato.

Se questa affermazione può risultare vera nel caso dei doni caritatevoli, non risulta altrettanto verificabile se applicata ai doni dei commercianti nei confronti del "Senza Monete". La solidarietà con gli obbiettivi dell'Associazione spinge a donare gratuitamente grazie ad una forte capacità di immedesimazione ed empatia: la generosità e la spontaneità del dono, la condivisione del fine ultimo del progetto eliminano il debito contratto dal ricevente, trasformando la relazione da dipendenza a cooperazione.

Un ultimo esempio di collaborazione dai tratti assimilabili alla logica del dono mi è stato fornito durante una lunga conversazione con una signora egiziana incontrata più volte al "Senza Monete". All'inizio della nostra prima conversazione la signora afferma "[...] a modo mio faccio anche un po' la volontaria. Sono egiziana, quando vado in Egitto porto cose, soprattutto per i bambini o per le famiglie che hanno difficoltà economiche, e lo faccio molto volentieri [...]». Quest'ultimo caso presenta delle caratteristiche ibride che legano le tipologie di dono fino ad ora incontrate: è una forma creativa di volontariato, dai tratti transnazionali, ideata per offrire supporto materiale nel proprio 
Paese di origine, investendo il tempo libero per cercare nell'Emporio oggetti di quotidiana utilità da donare in casi di particolare necessità.

Gli esempi qui proposti permettono di notare come il dono sia una modalità di scambio molto spesso utilizzata sia per le transazioni che avvengono in questa piccola realtà associativa sia negli scambi quotidiani al di fuori dell'Emporio.

\section{Conclusioni}

L'analisi e la descrizione di questo percorso etnografico mi hanno permesso di formulare alcune riflessioni sia sul consumo sia sullo scambio di cose: l'esame dei meccanismi che regolano il "Senza Monete" mostra come le transazioni che vi hanno luogo non siano riconducibili unicamente al baratto ma siano profondamente influenzate dal dono, categoria sempre più presente sia nella quotidianità dei singoli individui sia in gruppi più ampi ed organizzati. La piccola comunità che si è creata con gli anni attorno all'Associazione ha cercato, attraverso un pragmatismo solidale, di fornire delle risposte reali a molti individui e famiglie in stato di necessità.

51 Allo stesso tempo, l'eterogeneità dei soci è testimonianza di un pensiero critico nei confronti del consumo eccessivo e del consumismo sfrenato che cerca risposte alternative allo spreco, attraverso degli atteggiamenti ispirati al consumo critico ed ecologico. Questo gruppo composito e differenziato di volontari e soci che frequentano più o meno assiduamente l'Emporio offre una nitida panoramica di come la creatività umana si sia saputa attivare per trovare un'alternativa ad una crisi sociale ed economica sempre più avvertita.

Il "Senza monete", sebbene con le sue contraddizioni e con le sue peculiarità, si presenta come un caso paradigmatico ed emblematico: un luogo in cui dare una seconda possibilità agli oggetti, grazie al tempo donato volontariamente da un team di persone mosse da un obiettivo comune.

\section{BIBLIOGRAFIA}

Anderson B., 2018 Comunità immaginate. Origini e fortuna dei nazionalismi, Laterza, Roma.

Appadurai A., 2012 Modernità in polvere, Raffaello Cortina, Milano.

Appadurai A., 2013 Il futuro come fatto culturale. Saggi sulla condizione globale, Raffaello Cortina, Milano.

Appadurai A. (ed.), 1986 The social life of things. Commodities in Cultural Perspective, Cambridge University Press, Cambridge.

Aria M., 2016 I doni di Mauss. Percorsi di antropologia economica, CISU, Roma.

Aria M., Dei F. (a cura di), 2008 Culture del dono, Meltemi, Roma.

Bauman Z., 2010 Consumo dunque sono, Laterza, Roma. 
Cnaan R., Hunter A., Milofsky C., 2005 The Handbook of Community Movements and Local Organizations, Springer, New York.

Coluccia P., 2002 La cultura della reciprocità. I sistemi di scambio locale non monetari, Arianna Editrice, Casalecchio.

Dei F, Meloni P., 2015 Antropologia della cultura materiale, Carocci, Roma.

Douglas M., 2007 «Nessun dono è gratuito», in Equilibri, a. XI, vol. 3: 405-420.

Franzini M., 2013 Diseguaglianze inaccettabili, Laterza, Roma.

Godbout J. T., 2002 Lo spirito del dono, Bollati Boringhieri, Torino.

Grasseni C., Ronzon F., 2004 Pratiche e cognizione. Note di ecologia della cultura, Meltemi, Roma.

Gregory C., 1982 Gift and Commodities, Academic Press, Cambridge.

Hart K., 1982 «On Commodization», in E. Goody (ed.), From Craft to Industry: The Ethnography of Protoindustrial Cloth Production, Cambridge University Press, Cambridge: 38-49.

Hart K., 2009 «Money in the making of world society» in C. Hann, K. Hart (eds), Market and society. The great transformation today, Cambridge University press, Cambridge: 91-105.

Latouche S., 2008 Breve trattato sulla decrescita serena, Bollati Boringhieri, Torino.

Mauss M., 2002 Saggio sul dono. Forma e motivo dello scambio nelle società arcaiche, Einaudi, Torino.

Meloni P., 2018 Antropologia del consumo. Doni, merci, simboli, Carocci, Roma.

Miller D., 1987 Material Culture and Mass Consumption, Basil Blackwell, Oxford.

Miller D., 1998 Teoria dello Shopping, Editori Riuniti, Roma.

Miller D., 2012 Consumption and its Consequences, Polity, Cambridge.

Paltrinieri R., 1998 Il consumo come linguaggio, Franco Angeli, Milano.

Taylor M., 2010 «Community Participation», in K. Hart, J.L. Laville, A.D. Cattani (eds), The human economy. A citizen's guide, Polity Press, Cambridge: 236-247.

Taussig M., 2017 Il diavolo e il feticismo della merce. Antropologia dell'alienazione nel "patto col diavolo", Derive Approdi, Roma.

Torsello D., 2007 Dono, scambio e favore. Fondamenti e sviluppi dell'antropologia economica, Mondadori Università, Roma.

Weiner A., 1992 Inalienable Possessions. The Paradox of Keeping-While-Giving, University of California Press, Berkeley.

Weiner A. B., 1994 «Cultural difference and the density of objects», in American Ethnologist, XXI: 391-403. 


\section{ALLEGATO}

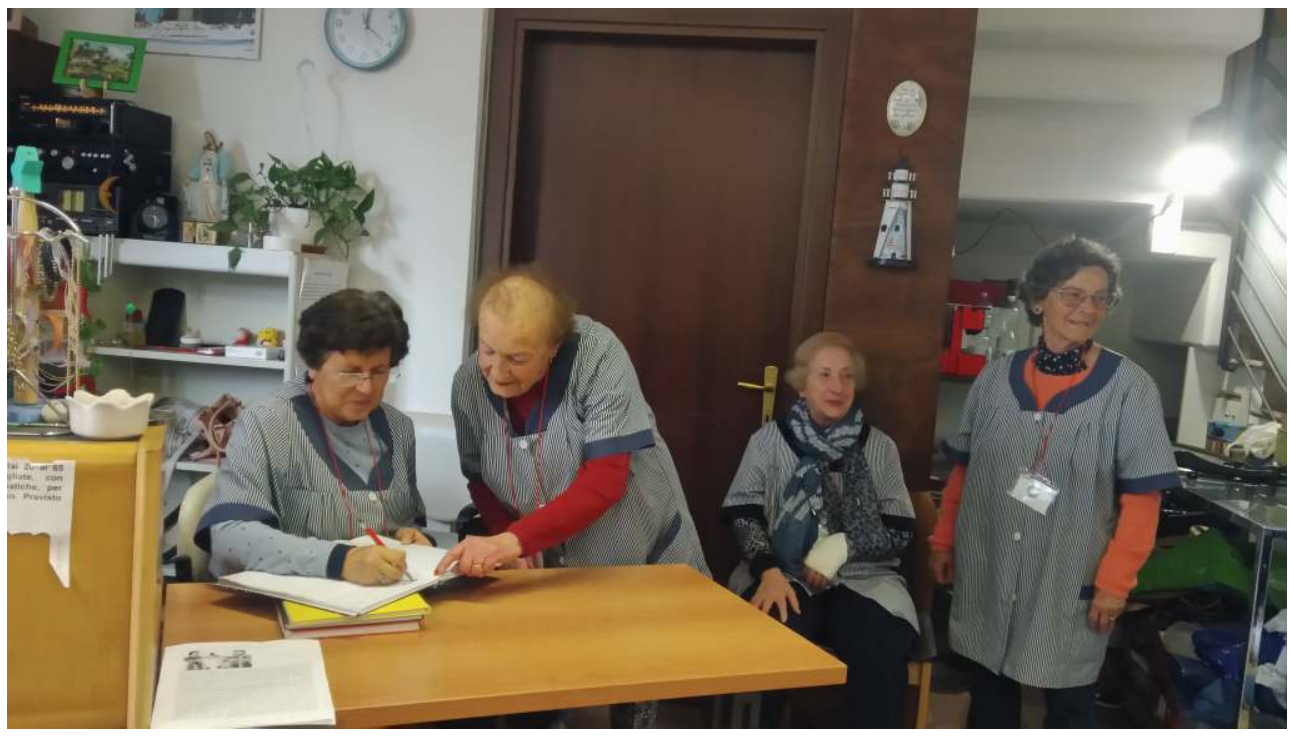

Foto 1. Le volontarie durante un momento di pausa

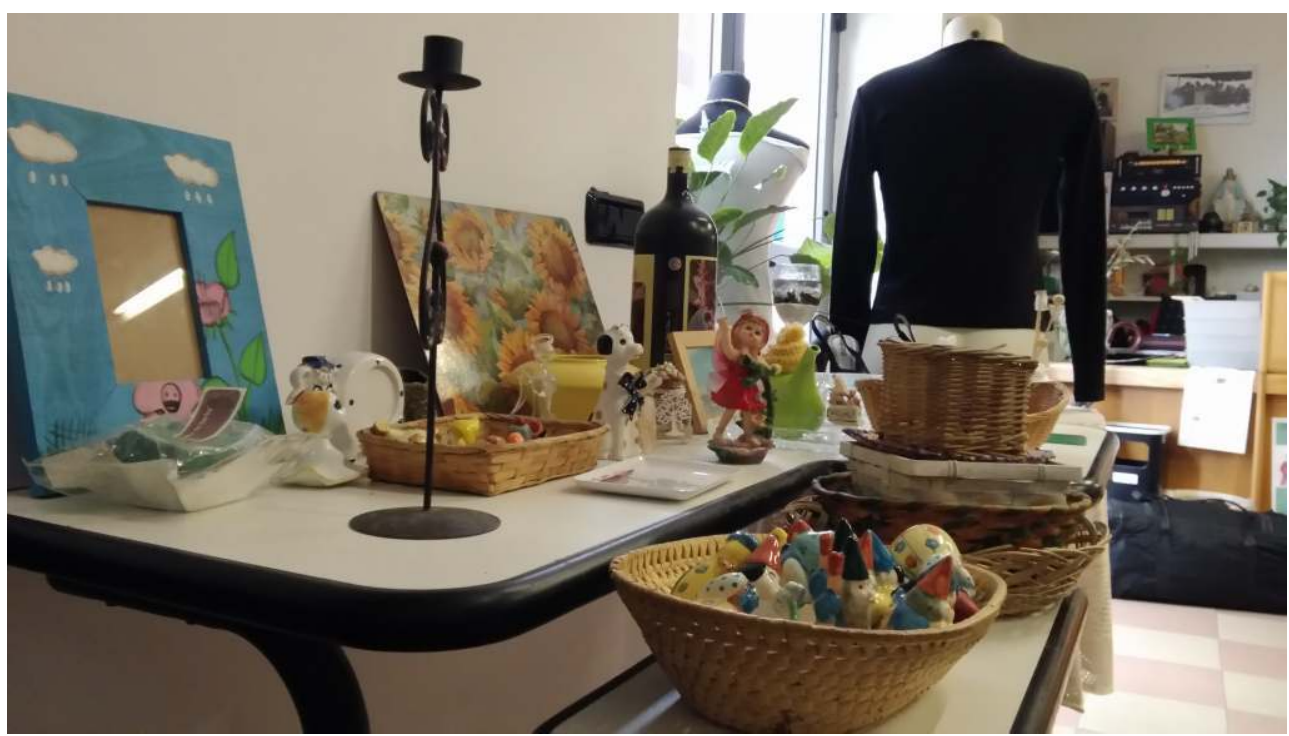

Foto 2. Gli oggetti considerati più pregiati e in migliori condizioni 


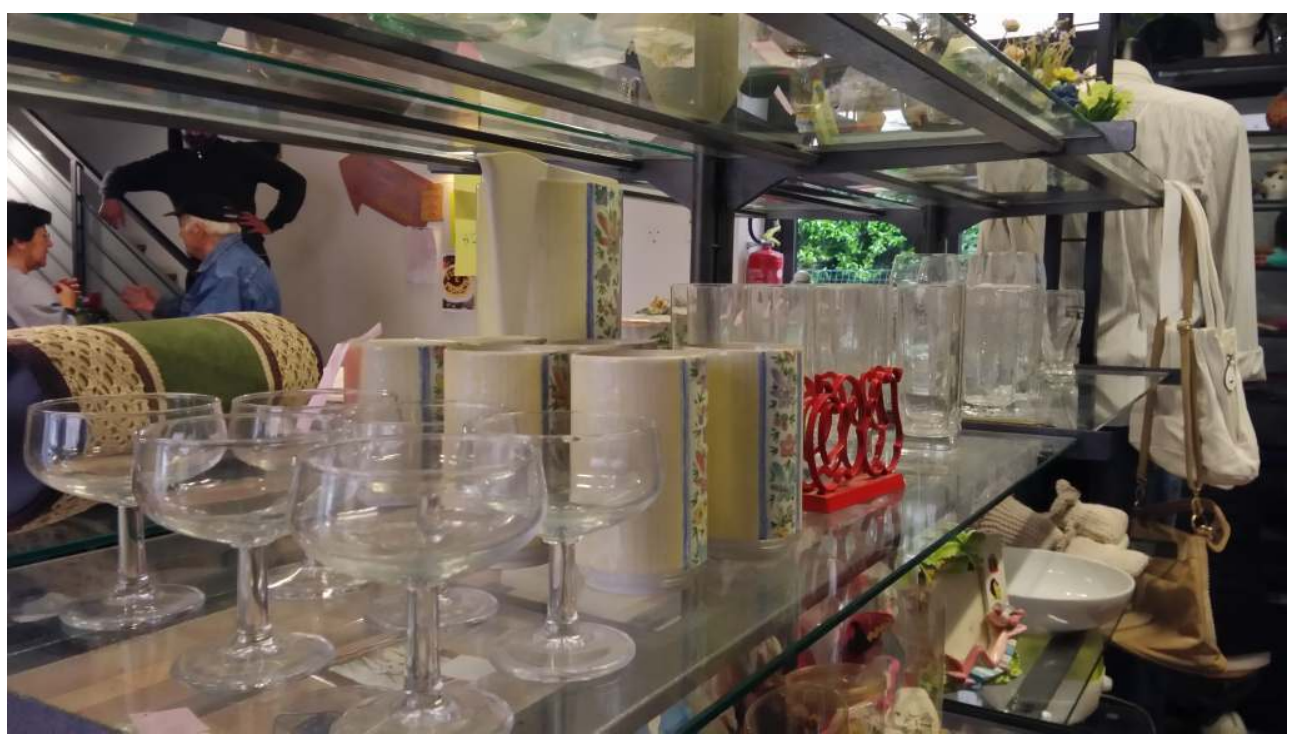

Foto 3. Set di bicchieri su un espositore al piano terra della Bottega

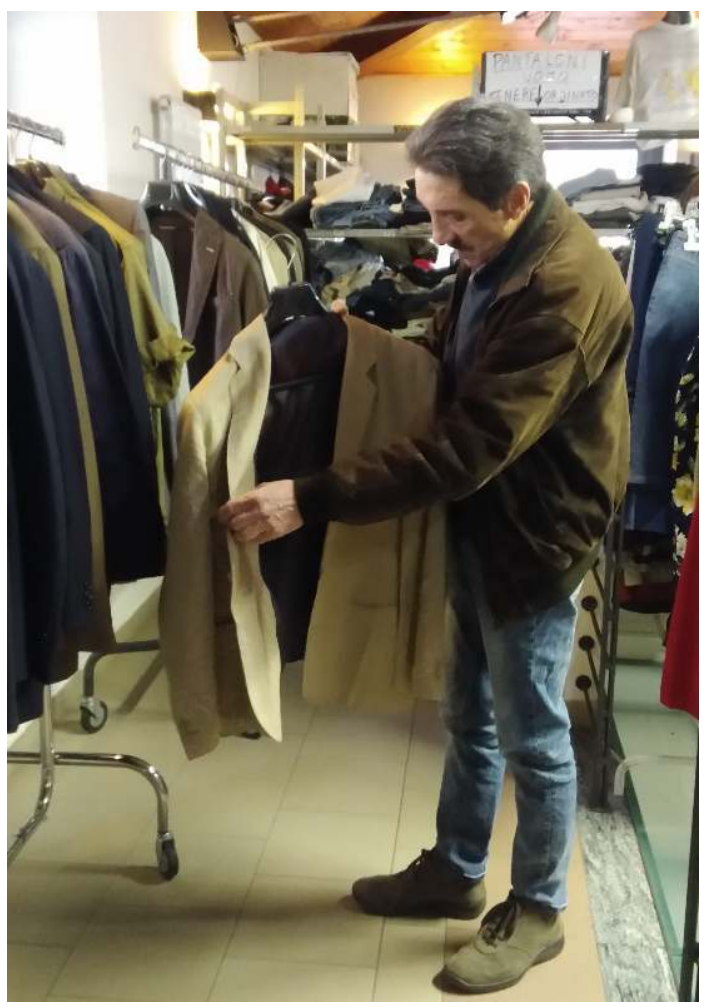

Foto 4. Un socio mentre controlla le condizioni di una giacca

\section{RIASSUNTI}

L'articolo è incentrato sull'analisi delle modalità di offerta e acquisizione di oggetti in un emporio dello scambio e del baratto, il "Senza Monete", situato ad Orvieto, in provincia di Terni. Attraverso interviste semi strutturate e un breve periodo di osservazione partecipante ho voluto proporre alcune riflessioni sul consumo, analizzando inoltre le modalità di circolazione delle merci all'interno di questa peculiare realtà associativa. I risultati emersi durante la ricerca mettono in luce dinamiche affini al dono, che talvolta esulano dal principio del baratto che regola 
la maggior parte degli scambi all'interno dell'Emporio. Le interviste rivolte ai soci hanno dimostrato una interessante eterogeneità negli approcci al consumo, sottolineando come una forte, talvolta contraddittoria, critica al consumismo regoli la maggior parte delle loro scelte, quando queste non sono dettate da necessità di natura economica. Il "Senza Monete" si presenta come un caso paradigmatico, un luogo in cui dare una seconda possibilità agli oggetti, grazie al tempo donato volontariamente da un team di persone che cercano di far fronte ad una sempre più avvertita crisi sociale ed economica.

The article focuses on the analysis of the methods of acquisition and supply of objects in an emporium of exchange and barter, the "Senza Monete", located in Orvieto, in the province of Terni. Through semi-structured interviews and participant observation, I would like to put forward some reflections on consumption and exchange by analyzing the ways of circulation of goods within this peculiar association reality. The results that emerged during the research highlight gift-related dynamics, which sometimes go beyond the barter principle that regulates most exchanges within the Emporium. The interviews addressed to the members have also shown an interesting heterogeneity within the process from approaching to consumption, demonstrating how a strong, sometimes contradictory, critic towards consumerism impacts most of their choices, when these are not dictated by financial needs. The "Senza Monete" presents itself as a paradigmatic case, a place that offers a second chance to objects thanks to the time donated voluntarily by a team of people who is trying to cope with an increasingly felt social and economic crisis.

\section{INDICE}

Keywords : gift, barter, consumption, exchange, goods

Parole chiave : dono, baratto, consumo, scambio, merci

\section{AUTORE}

\section{VIOLA LUCREZIA GIULIANI}

Dipartimento di Scienze Sociali, Politiche e Cognitive, Università degli Studi di Siena violucrezia10@gmail.com 